\title{
Hipertensión pulmonar idiopática en pediatría: Caso clínico y revisión de la literatura
}

\author{
ANA MARÍA HERRERA G.*, CLAUDIA ASTUDILLO M.** y MARÍA ANGÉLICA PALOMINO M.*
}

Idiopathic pulmonary hypertension in pediatric age: A case report and review

Idiopathic pulmonary hypertension is a rare disease in the pediatric age. Symptoms are subtle in first stages delaying diagnosis and resulting in worst outcomes. Therefore pediatricians should be aware of the initial symptoms. We report the case of a 13 years old girl, who started with progressive dyspnea 9 months before diagnosis. She was admitted to our hospital because of productive cough, fever and shortness of breath. Chest $X$ ray showed enlargement of right ventricle and pulmonary artery. Echocardiography was suggestive of pulmonary hypertension and the diagnosis was confirmed by cardiac catheterization. Work up for secondary causes of pulmonary hypertension was negative and pulmonary hypertension was considered idiopathic. Treatment with oxygen, diuretics, L-arginine, sildenafil, iloprost and acenocumarol was started with good clinical response.

Key words: Pulmonary hypertension, pulmonary vascular disease, sildenafil, prostacicline.

\section{Resumen}

La hipertensión pulmonar idiopática es de rara ocurrencia en la edad pediátrica siendo los síntomas iniciales muy sutiles e inespecíficos, lo que retarda el diagnóstico empeorando el pronóstico. Se presenta el caso de una niña 13 años, quien comienza con disnea progresiva 9 meses antes del diagnóstico. Ingresa por cuadro de tos húmeda, fiebre y dificultad respiratoria de 3 días de evolución. La radiografía de tórax muestra ventrículo derecho aumentado de tamaño y tronco de la arteria pulmonar prominente sospechándose una probable cardiopatía. Se realiza ecocardiografía que demuestra hipertensión pulmonar, la cual es corroborada por el sondeo cardíaco. Todos los exámenes de laboratorio fueron negativos para causas secundarias, estableciéndose el diagnóstico de hipertensión pulmonar idiopática. Se inicia manejo con oxígeno, diuréticos, L-arginina, sildenafil, iloprost nebulizado y acenocumarol, con lo cual la paciente evoluciona favorablemente.

Palabras clave: Hipertensión pulmonar, enfermedad vascular pulmonar, sildenafil, prostaciclina.

\section{Introducción}

La hipertensión pulmonar arterial de tipo idiopática es una condición caracterizada por elevación de presión arterial pulmonar sin causa demostrable. El criterio diagnóstico incluye un registro de presión arterial media $\geq 25 \mathrm{mmHg}$ en reposo o $\geq 30 \mathrm{mmHg}$ durante el ejercicio ${ }^{1}$. Inicialmente se utilizaba el término de hipertensión pulmonar primaria, pero actualmente se deno- mina hipertensión pulmonar arterial idiopática². Esta enfermedad es de carácter progresivo, presentándose más frecuentemente en mujeres jóvenes y de edad media ${ }^{3}$. Esta patología es de rara ocurrencia en niños y cuando se presenta está asociada habitualmente a cardiopatía congénita ${ }^{4}$. El pronóstico es ominoso cuando no se diagnostica y trata oportunamente. Los síntomas iniciales de la enfermedad son inespecíficos y sutiles y los hallazgos del examen físico tar-

* Pediatra Especialista en Enfermedades Respiratorias Infantiles, Departamento de Pediatría Norte, Facultad de Medicina, Universidad de Chile. Hospital Roberto del Río.

** Pediatra Becado Enfermedades Respiratorias Infantiles, Departamento de Pediatría Norte, Facultad de Medicina, Universidad de Chile. Hospital Roberto del Río. 


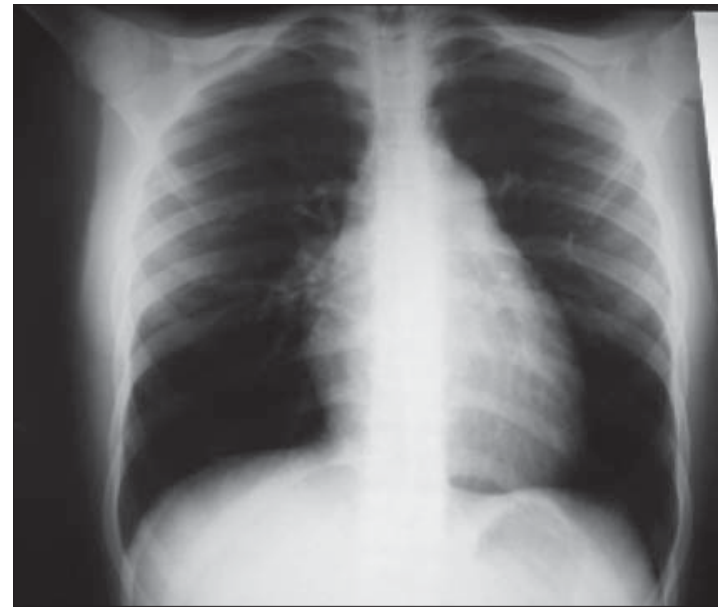

Figura 1. Radiografía de tórax AP.

díos, por lo que a menudo el diagnóstico se retrasa. El estudio está dirigido a descartar las causas secundarias de la enfermedad y a evaluar su gravedad. En los últimos años ha habido avances significativos en el conocimiento de la patogenia de esta enfermedad, lo que ha permitido establecer nuevas modalidades terapéuticas, como el surgimiento de fármacos tales como epoprostenol y bosentán lo que ha tenido un impacto significativo en el pronóstico y calidad de vida de estos pacientes ${ }^{5}$.

\section{Caso clínico}

Paciente de 13 años hospitalizada en diciembre del 2006 por cuadro de 3 días de tos húmeda, fiebre, dificultad respiratoria y requerimientos de oxígeno de 2 litros por minuto. Preguntando dirigidamente por síntomas que den cuenta del inicio de la enfermedad se encuentra la presencia de disnea de 9 meses de evolución, progresiva e invalidante. Un mes antes del ingreso refiere ortopnea, dolor torácico y cianosis, sin la presencia de síncopes. Al examen físico destaca apremio respiratorio, $2^{\circ}$ ruido cardíaco aumentado de intensidad y edema leve de extremidades inferiores. La radiografía de tórax muestra tronco de la arteria pulmonar prominente y ventrículo derecho aumentado de tamaño (Figuras 1 y 2). La ecocardiografía revela gran dilatación de ventrículo derecho el cual es de paredes hipertróficas y comprime al ventrículo izquierdo, forámen oval permeable con shunt de derecha a izquierda, insuficiencia tricuspídea moderada que permite estimar la presión de ar-

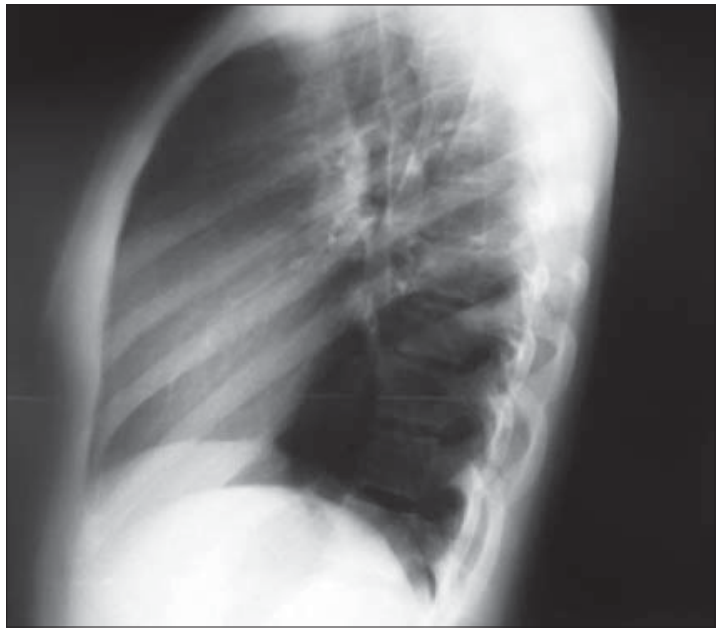

Figura 2. Radiografía de tórax lateral.

teria pulmonar en $110 \mathrm{mmHg}$. El sondeo cardíaco confirma presión pulmonar en nivel sistémico $(94 / 52 \mathrm{mmHg}$, presión media de arteria pulmonar $68 \mathrm{mmHg})$. Se realiza test de vasorreactividad con adenosina en dosis crecientes $(50,100,200$ $\mu \mathrm{g} / \mathrm{kg} / \mathrm{min}$ ) sin obtener cambio en la presión de arteria pulmonar. Por lo tanto, se concluye hipertensión pulmonar severa sin respuesta a vasodilatadores. Se descartan causas secundarias con distintos exámenes, los cuales resultan todos normales (gases arteriales, función pulmonar; cintigrama V/Q, angioTAC, ELISA VIH, ANCA, ENA, ANA, función hepática, renal y tiroidea). Se diagnostica hipertensión pulmonar arterial idiopática severa y se inicia tratamiento con oxígeno nocturno, espironolactona, L-arginina, acenocumarol y sildenafil. Además dos meses más tarde se agrega a la terapia iloprost nebulizado. Luego de 3 meses de tratamiento la paciente refiere sentirse mejor, sin disnea por lo que vuelve a asistir al colegio. El test de marcha de 6 minutos mejoró de 280 a 410 m caminados (35 a $51 \%$ del teórico). La paciente ha completado un año de tratamiento con buena respuesta, manteniéndose en capacidad funcional II a III.

\section{Discusión}

La arteria pulmonar es una estructura complaciente, lo que permite al lecho vascular pulmonar funcionar como un sistema de alto flujo y baja presión ${ }^{6}$. Normalmente la presión media de la arteria pulmonar en reposo es menor a $15 \mathrm{mmHg}$. Cuando ésta es mayor a 25 $\mathrm{mm}$ de $\mathrm{Hg}$ en reposo o mayor a $30 \mathrm{mmHg}$ con 
ejercicio se habla de hipertensión pulmonar. Esta enfermedad habitualmente es secundaria a una patología subyacente, lo más frecuente es su asociación con cardiopatías de corazón izquierdo y enfermedades parenquimatosas pulmonares. Cuando no puede establecerse la presencia de una enfermedad de base luego de un estudio acabado, podemos hacer el diagnóstico de hipertensión pulmonar idiopática (Tabla 1) 2,7,8. Se estima que la hipertensión pulmonar idiopática tiene una incidencia de 1 a 2 por millón de habi$\operatorname{tantes}^{6,9}$. Existe una forma familiar de la enfermedad, la cual tiene una herencia autosómica recesiva. Esta patología se produce por aumento de la resistencia vascular a nivel de la arteriola pulmonar, lo cual se produce por la combinación de tres mecanismos: vasoconstricción, remodelación de la pared vascular y trombosis in situ, siendo el segundo el más relevante ${ }^{10}$. La vasoconstricción obedece a un desbalance en el equilibrio que normalmente existe en el endotelio vascular, entre la producción local de sustancias vasoconstrictoras (tromboxano y endotelina) y las vasodilatadoras (óxido nítrico y prostaciclina) en favor de las primeras. Cuando la hipertensión en el territorio pulmonar se mantiene en el tiempo se produce una alteración anatómica de los vasos, con engrosamiento de la íntima y la media. Finalmente, se produce trombosis in situ por daño del endotelio vascular, fibrinolísis anormal, aumento de la actividad procoagulante y enlentecimiento del flujo sanguíneo en vasos que presentan estrechamiento de su lumen. Los síntomas inicialmente son muy sutiles e inespecíficos por lo que el diagnóstico se hace en promedio 2 años luego de iniciados los síntomas. El síntoma más frecuente es la presencia de disnea con ejercicio, presente en el $60 \%$ de los pacientes, porcentaje que se acerca casi al 100\% al progresar la enfermedad. Otros síntomas asociados son la presencia de dolor torácico, síncope y hemoptisis. Los hallazgos al examen físico son los asociados a sobrecarga del corazón derecho. Frente a un paciente con sospecha de hipertensión pulmonar se debe solicitar inicialmente una radiografía de tórax, la cual mostrará prominencia de las arterias pulmonares con oligohemia hacia la periferia y además hipertrofia del ventrículo derecho, como era el caso de nuestra paciente ${ }^{10}$. Sólo en $10 \%$ de los casos la radiografia de tórax es normal ${ }^{9}$. El electrocardiograma puede mostrar signos de hipertrofia ventricular derecha. El mejor instrumento de tamizaje disponible en la actualidad para el diagnóstico de hipertensión pulmonar es la ecocardiografía con doppler, ya que permite
Tabla 1. Clasificación de la hipertensión pulmonar ${ }^{2}$

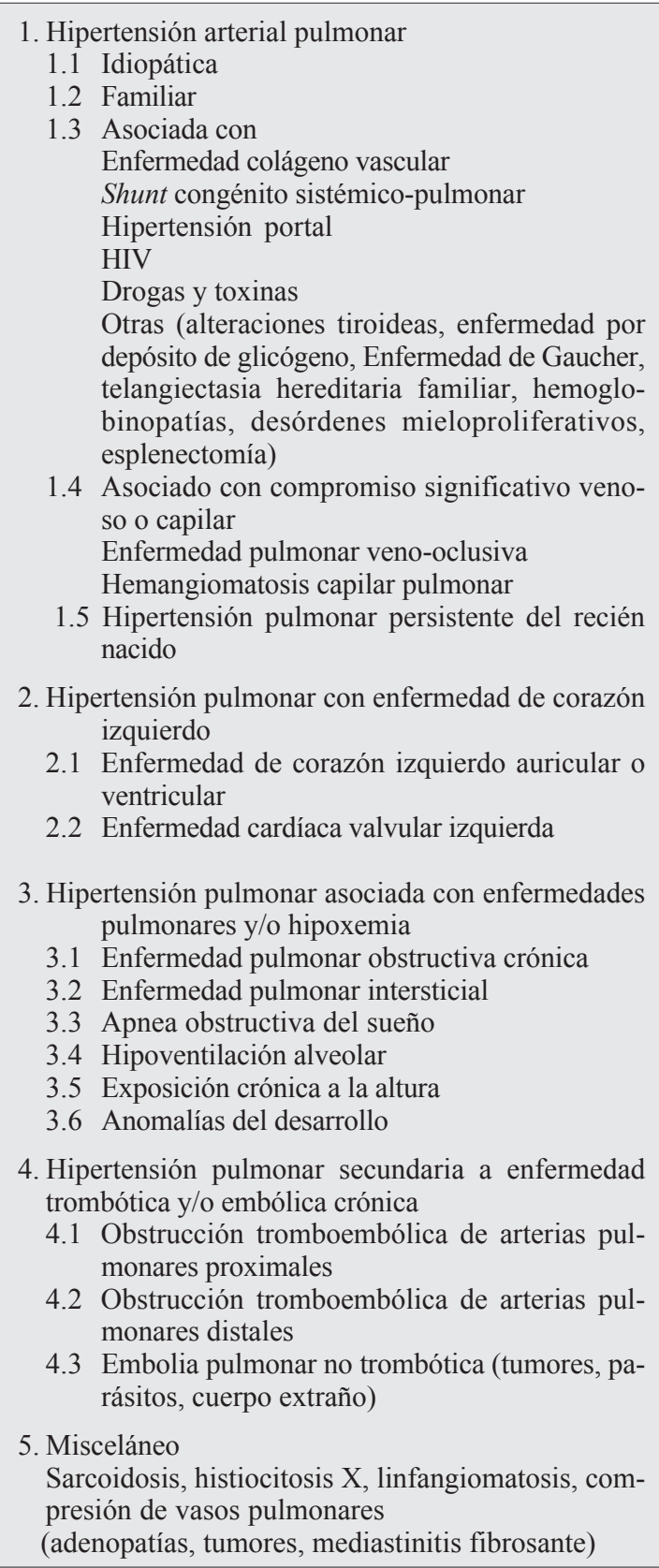

descartar la presencia de cardiopatía congénita y miocardiopatía y estimar la presión de la arteria pulmonar en base a la onda de regurgitación tricuspídea. Para establecer el diagnóstico de certeza se realiza cateterismo cardíaco, el cual permite además evaluar la respuesta a vasodilatadores como adenosina, prostaciclina u óxido nítrico (test de vasorreactividad). Éste se considera positivo cuando hay una caída de al menos $10 \mathrm{mmHg}$ en la presión media de la arteria 
pulmonar en relación al valor basal, debiendo llegar ademas a un valor absoluto igual o menor a $40 \mathrm{mmHg}^{11,12}$. Una vez establecido el diagnóstico de hipertensión pulmonar se debe descartar todas las posibles causas secundarias ${ }^{2,7,8}{ }^{13}$ (Tabla 1). El segundo paso es determinar la capacidad funcional del paciente ya que ésta se correlaciona directamente con el pronóstico y permite monitorizar el tratamiento ${ }^{14}$. Para objetivar la capacidad funcional que refiere el paciente se realiza el test de marcha de 6 minutos. La distancia caminada tiene excelente correlación con la severidad de la hipertensión pulmonar y es el mejor método disponible en la actualidad para evaluar la respuesta al tratamiento (evidencia tipo A). La hipertensión pulmonar idiopática es una enfermedad progresiva que no tiene cura. La sobrevida estimada es de 68 a $77 \%$ al año, 40 a $56 \%$ a los 3 años y 22 a $38 \%$ a los 5 años de seguimiento $^{14}$. El objetivo primario de la terapia no es disminuir la presión arterial pulmonar sino mejorar la capacidad funcional del paciente y prolongar su sobrevida. La hipertensión arterial pulmonar se modifica poco con el tratamiento, por lo que la evaluación de la respuesta al mismo no debe realizarse con mediciones seriadas de este índice sino con el test de marcha de 6 minutos. El tratamiento de los niños con hipertensión pulmonar idiopática es similar al de los adultos, pero los resultados son difíciles de predecir $^{10}$. La terapia básica consiste en la administración de oxígeno en los pacientes que tienen saturaciones menores a $91 \%^{10,14}$, ya que bajo este valor se produce vasoconstricción del territorio pulmonar. Para disminuir la precarga del ventrículo derecho se puede utilizar diuréticos ${ }^{14}$ como la espironolactona. Los pacientes con hipertensión pulmonar desarrollan trombosis in situ, importante factor de progresión de la enfermedad, por esta razón se recomienda el uso de terapia anticoagulante oral que mantenga INR $=1,5$ y $2,5^{14}$. Uno de los pilares del tratamiento de la hipertensión pulmonar es el uso de distintas familias de vasodilatadores, como los bloqueadores de los canales de calcio. Estos últimos sólo están indicados en el 10\% de los pacientes con hipertensión pulmonar que cursan con test de vasorreactividad positivo. En estos pacientes los bloqueadores de los canales de calcio pueden mejorar la sobrevida ${ }^{14-16}$. En niños que responden al test de vasorreactividad el uso de bloqueadores de canales de calcio mejora la calidad de vida y la sobrevida a 5 años $(97 \%$ versus $35 \%$ en los no respondedores $)^{8}$. En el ultimo tiempo han surgido nuevos fármacos que tienen acción principalmente antiproliferativa, lo que conduce a una remodelación de los vasos alterados, disminuyendo la resistencia vascular pulmonar con mejoría del gasto cardíaco. Un ejemplo de esto son los análogos de prostaciclina o prostanoides. La prostaciclina es un metabolito del ácido araquidónico producido normalmente en el endotelio vascular. Esta sustancia es un potente vasodilatador pulmonar y sistémico y tiene además efecto antiagregante plaquetario. Los prostanoides estimulan los receptores de prostaciclina produciendo vasodilatación del territorio pulmonar, inhibiendo el crecimiento de fibras musculares lisas y la agregación plaquetaria. Estos medicamentos pueden ser administrados por distintas vías: endovenoso (epoprostenol), subcutáneo (treprostinil), vía oral (beraprost) y nebulizado (iloprost). El epoprostenol (Flolan R) es en la actualidad el tratamiento de elección para los pacientes con capacidad funcional III a IV refractarios a otras terapias. Es de vida media corta, 1 a 2 minutos, por lo que debe administrarse en infusión continua y a través de catéter venoso central por su efecto irritante en las venas periféricas ${ }^{10}$. En adultos este medicamento disminuye la resistencia vascular pulmonar mejorando el test de marcha luego de 12 semanas de tratamiento y la sobrevida a 5 años (55\% versus $28 \%$ en el grupo control) $)^{17,18}$. En niños el uso de prostaciclina endovenosa ha sido también exitoso con una sobrevida a 5 años de $92 \%$ en comparación con $29 \%$ en aquellos en que el medicamento no estuvo disponible ${ }^{8}$. Epoprostenol no está ampliamente distribuido en nuestro país, pero se puede conseguir para casos especiales. El iloprost nebulizado (Ventavis R) está disponible en Chile desde 2005 y al igual que epoprostenol tiene una vida media corta de 20 a 25 minutos, por lo que su uso debe ser repetido a lo largo del día (6 a 9 nebulizaciones diarias). Su uso está recomendado para pacientes con capacidad funcional III, en los cuales el medicamento produce mejoría sintomática, funcional y hemodinámica ${ }^{11,19}$. Otra familia de vasodilatadores corresponde a los antagonistas de los receptores de endotelina. La endotelina-1 es un potente péptido vasoactivo producido por las células del endotelio vascular cuya expresión está aumentada en los pacientes con hipertensión pulmonar idiopática ${ }^{13}$. Produce vasoconstricción, estimula la proliferación de células musculares lisas y actúa como co-mitógeno induciendo fibrosis. Los efectos de la endotelina-1 son mediados a través de los receptores de endotelina A y B. La activación de los receptores de endotelina A causa vasoconstricción y proliferación de células musculares lisas, mientras que la de 
endotelina $\mathrm{B}$ induce la producción de óxido nítrico y prostaciclina. Bosentan es un antagonista no selectivo de los receptores de endotelina A y $\mathrm{B}^{14}$, siendo actualmente considerado la primera elección para pacientes con capacidad funcional II y III. Actualmente está en tramitación la licencia para la venta de este producto en Chile. Se ha comunicado que el uso de este medicamento produce disminución de la presión arterial pulmonar, mejoría significativa de la distancia caminada, índice de disnea y capacidad funcional, alargando además el tiempo libre de deterioro clínico ${ }^{13,14,20}$. En niños de 4 a 17 años existe un estudio que demuestra que bosentan es capaz de disminuir significativamente la presión y el índice de resistencia vascular pulmonar ${ }^{21}$. Actualmente se han desarrollado bloqueadores selectivos de los receptores A de endotelina como el ambrisentan, ya aprobado por la FDA y sitaxsentan en espera de aprobación. Estos bloqueadores selectivos eliminan los efectos indeseables de la activación de los receptores A de endotelina (vasoconstricción) y dejan los efectos beneficiosos de los receptores B (vasodilatación $)^{14}$. Otra estrategia para producir vasodilatación en el territorio pulmonar es aumentar la cantidad de óxido nítrico endógeno, potente vasodilatador. El sildenafil actúa bloqueando a la fosfodiesterasa 5, enzima que inhibe al cGMP, segundo mensajero del óxido nítrico, con lo que aumenta este último produciéndose vasodilatación. Sildenafil tendría además acción antiproliferativa $^{12}$. Con el uso de este medicamento se ha comunicado aumento del tiempo de ejercicio, disminución de la presión sistólica pulmonar y mejoría del grado de disnea y fatiga ${ }^{22}$. En un estudio multicéntrico, doble ciego, placebo controlado que incluyó 278 pacientes con hipertensión pulmonar sintomática se demostró que el uso de sildenafil mejora la capacidad de ejercicio, la capacidad funcional y la hemodinamia ${ }^{23}$. La eficacia de este medicamento también ha sido comunicada en niños, pero sólo en pequeñas series de $\operatorname{casos}^{10,24}$ en las cuales su uso mostró ser beneficioso. Actualmente se estaría llevando a cabo un estudio randomizado en pacientes pediátricos. La gran ventaja de sildenafil es ser el medicamento más ampliamente disponible en Chile con la mejor relación costo-efectividad. Con el mismo objetivo de aumentar la producción endógena de óxido nítrico es que se ha utilizado la L-arginina, único sustrato de la óxido nítrico sintetasa. Se ha visto que la suplementación oral con L-arginina por más de 1 semana en pacientes con hipertensión pulmonar produce mejoría significativa de la hemodinamia pulmonar y del consumo máximo de oxígeno en el test de ejercicio ${ }^{25}$. Al parecer la mejor estrategia de tratamiento es el uso combinado de los medicamentos disponibles en la actualidad, los que pueden actuar a distinto nivel, aprovechando el efecto sinérgico de algunos de ellos ${ }^{25}$. En pacientes graves, refractarios a la terapia médica óptima, que presentan síncope recurrente e insuficiencia cardíaca derecha grave, se ha reportado el uso de la septostomía atrial. Esta consiste en la creación de un shunt interauricular para disminuir la presión de llene y la sobrecarga de volumen del corazón derecho. Es importante considerar que este procedimiento se utiliza como medida de salvataje en espera de trasplante pulmonar y que además es de altísimo riesgo por agravamiento de la hipoxia ${ }^{7,13}$. El trasplante pulmonar se reserva para pacientes con capacidad funcional III a IV que presenten deterioro clínico a pesar de recibir terapia médica óptima que incluya el uso de epoprostenol endovenoso $^{10,11}$. La sobrevida de pacientes que reciben trasplante es de $65 \%$ el primer año y $45 \%$ a los 5 años ${ }^{10}$, cifras que son inferiores a las de pacientes que reciben tratamiento médico con epoprostenol ${ }^{9}$. Por otro lado, la mortalidad del trasplante pulmonar es significativamente mayor en pacientes con hipertensión pulmonar en comparación con pacientes trasplantados por otra causa ${ }^{26}$. El trasplante pulmonar se indica sólo en el 14 a $17 \%$ de los pacientes pediátricos ${ }^{13}$ $y$ en el $6 \%$ de los adultos, lo que refleja que el tratamiento médico sería el de elección. Los pacientes se deben enlistar cuando la probabilidad de sobrevida a 2 años sea menor a $50 \%{ }^{5}$. Existe consenso que el trasplante bipulmonar es el de mejor pronóstico ${ }^{8}$ y que sólo por escasez de órganos algunos centros hacen monopulmonar. El trasplante cardiopulmonar se reserva para pacientes con cardiopatía congénita irreparable o de muy difícil corrección.

En conclusión podemos decir que la hipertensión pulmonar idiopática es una patología cuyo diagnóstico requiere un alto índice de sospecha por lo sutil de los síntomas. El mejor método de tamizaje es la ecocardiografía con doppler y para confirmar el diagnóstico el cateterismo cardíaco. El tratamiento médico es la mejor alternativa disponible actualmente para el manejo de estos pacientes, especialmente si se usa epoprostenol.

\section{Agradecimientos}

Agradecemos a la Dra. Mónica Zagolín, neumólogo de adultos a cargo del programa de 
hipertensión pulmonar del Instituto Nacional del Tórax por su constante apoyo y orientación en el manejo de nuestra paciente.

\section{Bibliografía}

1.- ROSENZWEIG E, BARST R. Idiopathic pulmonary arterial hypertension in children. Current Opinion in Pediatrics 2005; 17: 372-80.

2.- SIMONNEAU N, GALIE N, RUBIN L, LANGLEBEN D, SEEGER W, DOMENIGHETTI G, et al Clinical classification of pulmonary hypertension. J Am Coll Cardiol 2004; 43(12 suppl): s5-s12.

3.- SÁENZ C, SÁNCHEZ V, VELÁZQUEZ M T, TELLO R, GÓMEZ M, DELGADO J, et al. Guías de práctica clínica de la Sociedad Española de Cardiología en tromboembolismo e hipertensión pulmonar. Rev Esp Cardiol 2001; 54: 194-210.

4.- LANG I, BONDERMAN D, KNEUSSL M, MARX M Paediatric pulmonary vascular disease. Ped Resp Rev 2004; 5: 238-48.

5.- ROSENZWEIG E B, WIDLITZ A C, BARST R S. Pulmonary arterial hypertension in children. Pediatr Pulmonol 2004; 38: 2-22.

6.- GAINE S P, RUBIN L J. Primary pulmonary hypertension. Lancet 1998; 352: 719-25.

7.- IVY D. Diagnosis and treatment of severe pediatric pulmonary hypertension. Cardiology in Review 2001; 9: 227-37.

8.- BARST R. Recent advances in the treatment of pediatric pulmonary arterial hypertension. Ped Clin North Am 1999; 46: 331-45.

9.- ZAGOLÍN M B, WAINSTEIN E G, URIARTE P G. Actualización en el diagnóstico y terapéutica en hipertensión pulmonar arterial. Rev Méd Chile 2006; 134: $902-9$.

10.- DONTI A, FORMIGARI R, RAGNI L, MANES A, GALIE N, PICCHIO F, Pulmonary arterial hypertension in pediatric age. J Cardiovasc Med 2007; 8: 72-7

11.- RUBIN L J. Pulmonary arterial hypertension. Proc Am Thorac Soc 2006; 3: 111-5.

12.- HOEPER M M, RUBIN L. Update in pulmonary hypertension 2005. Am J Respir Crit Care Med 2006; 173: 499-505.

13.- RASHID A, IVY D. Severe paediatric pulmonary hypertension: new management strategies. Arch Dis Child 2005; 90: 92-8.

14.- HUMBERT M, SITBON O, SIMONNEAU G.
Treatment of pulmonary arterial hypertension. N Engl J Med 2004; 351: 1425-36.

15.- RICH S, KAUFMAN E, LEVY P S. The effect of high doses of calcium-channel blockers on survival in primary pulmonary hypertension. N Engl J Med 1992; 327: $76-81$.

16.- SITBON O, HUMBERT M, JAIS X, IOOS V, HAMID A, PROVENCHER S, et al. Long-term response to calcium channel blockers in idiopathic pulmonary arterial hypertension. Circulation 2005; 111: 310511.

17.- BARST R, RUBIN L, LONG W, MCGOONM, RICH $\mathrm{S}$, BADESCH D, et al. A comparison of continuous intravenous epoprostenol (prostacyclin) with conventional therapy for primary pulmonary hypertension. New Engl J Med 1996; 334: 296-302.

18.- SITBON O, HUMBERT M, NUNES H, PARENT F, GARCÍA G, HERVÉ P, et al. Long-term intravenous epoprostenol infusion in primary pulmonary hypertension: prognostic factors and survival. J Am Coll Cardiol 2002; 40: 780-8.

19.- OLSCHEWSKI H, SIMONNEAU G, GALIE N, HIGENBOTTAM T, NAEIJE R, RUBIN L, et al. Inhaled iloprost for severe pulmonary hypertension. New Engl J Med 2002; 347: 322-9.

20.- RUBIN L, BADESCH D, BARST R, GALIE N, BLACK $\mathrm{C}, \mathrm{KEOGH} A$, et al: Bosentan therapy for pulmonary arterial hypertension. N Engl J Med 2002; 346: 896903.

21.- BARST R J, IVY D, DINGEMANSE J, WIDLITZ A, SCHMITT K, DRAN A, et al. Pharmacokinetics, safety and efficacy of bosentan in pediatric patients with pulmonary artery hypertension. Clin Pharmacol Ther 2003; 73: 372-82.

22.- SASTRY B, NARASIMHAN C, REDDY K, RAJU S. Clinical efficacy of sildenafil in primary pulmonary hypertension. J Am Coll Cardiol 2004; 43: 1149-53.

23.- GALIE N, GHOFRANI H, TORVICKY A, BARST R, RUBIN L, BADESCH D, et al. Sildenafil citrate therapy for pulmonary arterial hypertension. New Engl J Med 2005; 353: 2148-57.

24.- LEIBOVITCH L, MATOK I, PARET G. Therapeutic application of sildenafil citrate in the management of paediatric pulmonary hypertension. Drugs 2007; 67: 57-73.

25.- HOWARD L, MORREL N. New therapeutic agents for pulmonary vascular disease. Paed Resp Rev 2005; 6: 285-91.

26.- RADLEY-SMITH R, AURORA P. Transplantation as a treatment for end-stage pulmonary hypertension in childhood. Paed Resp Rev 2006; 7: 117-22.

\footnotetext{
Correspondencia a:

Dra. Ana María Herrera G.

Pediatra Especialista en Enfermedades

Respiratorias Infantiles, Departamento de

Pediatría Norte, Facultad de Medicina,

Universidad de Chile. Hospital Roberto del Río.

E-mail: amherrerag@yahoo.com
} 